\title{
Eigenspine: Computing the Correlation between Measures Describing Vertebral Pose for Patients with Adolescent Idiopathic Scoliosis
}

\author{
Daniel Forsberg ${ }^{\mathrm{a}, \mathrm{c}}$, Claes Lundström ${ }^{\mathrm{a}, \mathrm{c}, *}$, Hans Knutsson ${ }^{\mathrm{b}, \mathrm{c}}$ \\ ${ }^{a}$ Sectra, Linköping, Sweden \\ ${ }^{b}$ Department of Biomedical Engineering, Linköping University, Sweden \\ ${ }^{c}$ Center for Medical Image Science and Visualization (CMIV), Linköping University, Sweden
}

\begin{abstract}
This paper describes the concept of eigenspine, a concept applicable for determining the correlation between pair-wise combinations of measures useful for describing the three-dimensional spinal deformities associated with adolescent idiopathic scoliosis. The proposed data analysis scheme is based upon the use of principal component analysis (PCA) and canonical correlation analysis (CCA). PCA is employed to reduce the dimensionality of the data space, thereby providing a regularization of the measurements, and CCA is employed to determine the linear dependence between pair-wise combinations of different measures. The usefulness of the eigenspine concept is demonstrated by analyzing the position and the rotation of all lumbar and thoracic vertebrae as obtained from 46 patients suffering from adolescent idiopathic scoliosis. The analysis showed that the strongest linear relationship is found between the lateral displacement and the coronal rotation of the vertebrae, and that a somewhat weaker but still strong correlation is found between the coronal rotation and the axial rotation of the vertebrae. These results are well in-line with the general understanding of idiopathic scoliosis. Noteworthy though is that the correlation between the anterior-posterior displacement and the sagittal rotation was not as strong as expected and that the obtained results further indicate the need for including the axial vertebral rotation as a measure when characterizing different types of idiopathic scoliosis. Apart from analyzing pair-wise correlations between different measures, the method is believed to be suitable for finding a maximally descriptive low-dimensional combination of measures describing spinal deformities in idiopathic scoliosis.
\end{abstract}

Keywords: Spine imaging, principial component analysis, canonical correlation analysis, pose estimation, idiopathic scoliosis

\section{Introduction}

Scoliosis is a disease that affects the spine by introducing an excessive lateral curvature of the spine, as can be observed by examining the asymmetry of a person's back or as is evident on e.g. an anterior-posterior radiograph, commonly employed when setting the diagnosis of scoliosis. A scoliotic curvature is typically defined as a lateral curvature of the spine, where the Cobb angle exceeds $10^{\circ}$, as defined by the Scoliosis Research Society [1]. There are different types of scoliosis with various underlying causes, e.g. congenital scoliosis, neuromuscular scoliosis, degenerative scoliosis (induced by instabilities caused by trauma, bone collapse, osteoporosis or previous back surgery) and idiopathic scoliosis, and where the latter is the most common type. Of the different types of scoliosis, adolescent idiopathic scoliosis (AIS) is the most frequently occurring type, and is, therefore, in the main focus of this paper.

AIS is typically detected during a standard physical examination, performed by a general practitioner or a school nurse. The diagnosis scoliosis is not given, though, until an anterior-

\footnotetext{
${ }^{*}$ Corresponding author

Email addresses: daniel.forsberg@sectra.se (Daniel Forsberg), claes.lundstrom@sectra.se (Claes Lundström),

hans.knutsson@liu.se (Hans Knutsson)
}

posterior radiograph has been used to measure the Cobb angle [2], and where the Cobb angle exceeds $10^{\circ}$. The Cobb angle is defined as the angle between two lines drawn parallel to the superior endplate of the superior end vertebra and parallel to the inferior endplate of the inferior end vertebra, illustrated in Figure 1. The end vertebrae of a curve are defined as the vertebrae that are the most tilted in the curve, as observed in the coronal plane. In addition, the diagnosis idiopathic scoliosis includes ruling out any other causes of scoliosis, since idiopathic scoliosis is a diagnosis of exclusion [3, 4].

Treatment of AIS is dependent on a number of factors, which include age of onset, gender, skeletal maturity, the Cobb angle and the estimated progression rate of the scoliosis [5]. Patients with a Cobb angle of less than $25^{\circ}$ (mild scoliosis) are in general recommended physical therapy and observation at regular intervals in order to monitor a possible progression. If the Cobb angle exceeds $25^{\circ}$ but is less than $40^{\circ}$ (moderate scoliosis) and the patient has remaining growth, then bracing is usually offered as treatment. The aim of the bracing is to prevent further progression until full skeletal maturity has been reached, at which the risk for further progression and the need for surgery greatly diminishes. In addition, bracing will hopefully correct some of the already induced deformation. For more severe cases of scoliosis, e.g. a Cobb angle larger than $45^{\circ}$ or a high progression rate, then surgery is necessary in order both 


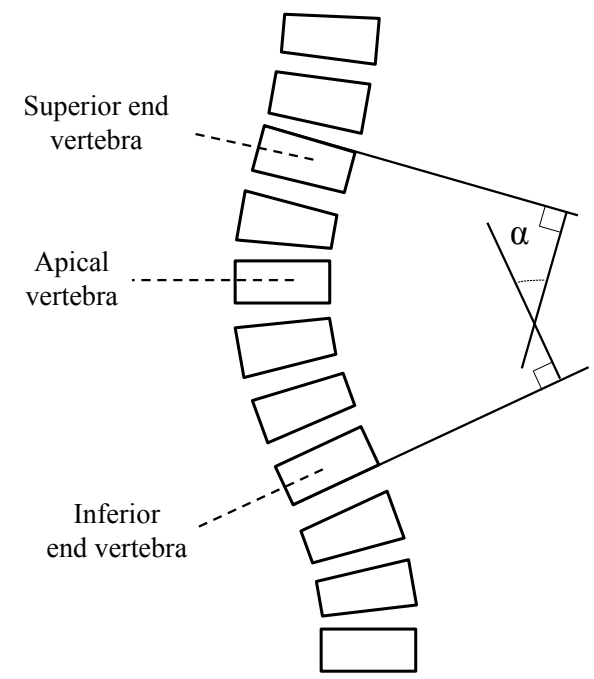

Figure 1: Schematic example of the Cobb angle, measured as the angle between the two lines drawn parallel to the upper and the lower endplates of the two end vertebrae in the scoliotic curvature.

to stop the progression and to correct some of the deformation. In surgery, a fusion is performed, a procedure where hooks, rods and screws are attached to the curved part of the spine and employed to straighten the spine, always including the primary scoliotic curve and sometimes also the secondary compensatory curve.

An important aspect for scoliosis in general, and in particular for idiopathic scoliosis, is how to assess the degree of scoliosis, i.e. the severity, since this is an integral component for deciding upon treatment, for observing the progression of the disease and for measuring the outcome of a treatment. As previously noted, scoliosis is generally considered as an excessive lateral curvature, hence, most measures related to deformity assessment attempt to quantify the degree of scoliosis by various measures in the coronal plane using an anterior-posterior radiograph. A review of commonly employed measures are found in [6], where the Cobb angle is the most frequently employed measure.

The Cobb angle plays, as already described, an important role in idiopathic scoliosis. However, although an established measure, the Cobb angle measures a two-dimensional (2D) projection of what is actually a three-dimensional (3D) deformity, and, therefore, the relevance of using the Cobb angle alone for assessment of the spinal deformity of a scoliotic curvature can be questioned. The 3D nature of the spinal deformity induced by scoliosis can be observed in Figure 2. Based upon the actual 3D deformity and the unknown etiology of idiopathic scoliosis, recent research has shown an interest in obtaining other measures for describing and quantifying a scoliotic deformity, where especially axial vertebral rotation (AVR) has received a great deal of interest [7, 8, 9].

Measuring the AVR can be done in a number of ways using manual methods, using either conventional radiography or 3D data as obtained from either CT or MR imaging. Different manual methods are discussed in $[10,11]$. Manual meth- ods, however, often suffer from being time-consuming, complex and related with a relatively high intra- and inter-observer variability, something which calls for computerized methods. In response to the need for computerized methods, a number of semi-automic methods for measuring the AVR have been proposed. These include the suggestions by Rogers et al. [12], Adam and Askin [13], Kouwenhoven et al. [14], Vrtovec [15] and our own work in [16]. The three first referred methods are limited in measurement accuracy, since they only use 2D axial images when estimating the rotation, whereas the methods in $[15,16]$ perform the measurements in $3 \mathrm{D}$. Note that the latter two methods not only measure the AVR, but the full pose of each vertebra, i.e. both position and rotation.

Another approach for assessing the scoliotic curvature, and which is not limited to measuring the AVR, is to use stereoradiography and reconstruction techniques for $3 \mathrm{D}$ modeling of the vertebrae. Brown et al. [17] introduced this approach and recent work is mostly related to the commercially available EOS system [18, 19, 20, 21].

Apart from developing methods that can provide a more accurate $3 \mathrm{D}$ description of spinal deformities, there is also a need to analyze how different measures describing spinal deformities relate to each other and to the clinical outcome [22, 23, 24]. This is important in order to classify various sub-types of idiopathic scoliosis and to determine if different treatments are suitable for the different sub-types of idiopathic scoliosis. Examples of this kind of work are found in [25, 26, 27], where they apply clustering algorithms to the measures derived from the EOS system [21], in order to identify various sub-types of idiopathic scoliosis. Another interesting and very recent work is found in [30], where the authors propose a global geometric torsion index for distinguishing between different classes of idiopathic scoliosis.

However, thus far, there has been limited amount of work performed, aimed at analyzing the relation between different measures relevant for describing a spinal deformity. To this end, we present the concept of eigenspine, a data analysis scheme for analyzing the correlation between different measures relevant for describing spinal deformities (e.g. vertebral pose) in idiopathic scoliosis. A novel aspect of our approach is that we analyze the linear relationship between sets of measurements of various measures. For example, the correlation between the coronal rotation of the vertebra and lateral displacement of the vertebra is not assessed only between single vertebrae but for the vertebrae of the whole spinal curvature. It is our belief that the knowledge about which measures that are the most relevant for describing a spinal deformity in (adolescent) idiopathic scoliosis provides new opportunities for establishing new clinical classification schemes, taking the full 3D deformity into account.

\section{Method - Eigenspine}

The proposed data analysis scheme is based on a combination of principal component analysis (PCA) [31] and canonical correlation analysis (CCA) [32], where PCA is used to reduce 

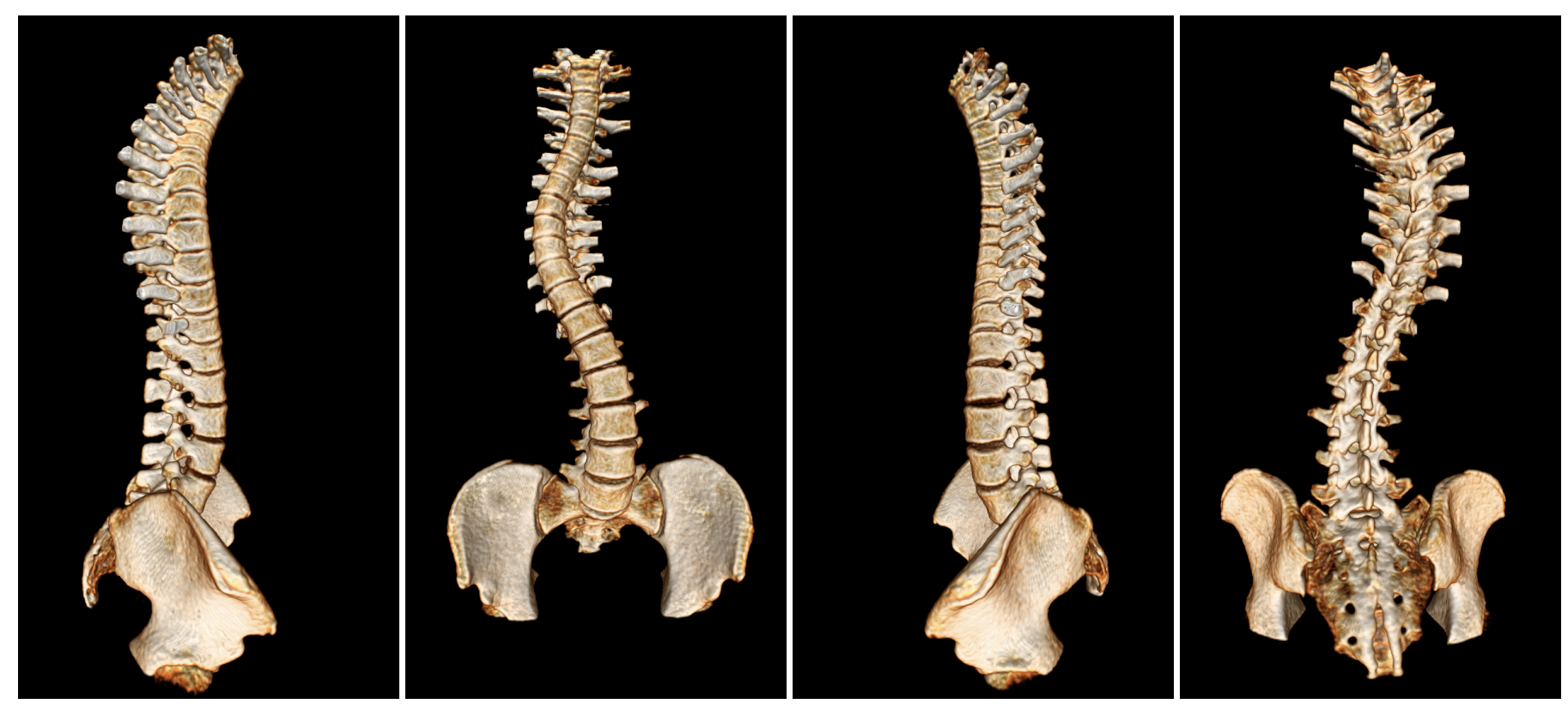

Figure 2: A 3D visualizations of a scoliotic spine clearly reveal the 3D nature of the deformity at hand, i.e. not only a lateral displacement of the spine but also a rotation and deformation of the individual vertebrae. In this case, the CT data has been visualized from the right, the front, the left and the back of a patient.

the number of dimensions in the measurement space, i.e. a regularization, and CCA is used to determine the linear dependence between pair-wise combinations of the different measures. For example, given a set of measurements of a number of measures for each vertebra in the spine, PCA is first employed on each measure to reduce the dimensionality of the respective measures. Thereafter, CCA is employed to determine the pairwise correlation between the different measures. The term eigenspine is used, since both the PCA and the CCA are performed by solving an eigenvector problem.

In this work, although the proposed scheme is primarily employed to analyze the linear dependence between various measures, the long-term goal of the analysis is to determine which measures, or combination of measures, that are significant for describing and assessing a scoliotic curvature. Thereby providing an approach for creating a classification scheme similar to the ones that are typically used, e.g. King and Lenke [28, 29], but in this case relying on a 3D description of the deformity, instead of merely using 2D projections of a 3D deformity.

\subsection{PCA and CCA}

PCA and CCA are two standard techniques for exploring data and is typically applied in unsupervised learning. For the sake of completeness, we will briefly introduce the two methods. Let $\mathrm{X}$ denote a data matrix

$$
\mathrm{X}=\left[\begin{array}{llll}
\mathbf{x}_{1} & \mathbf{x}_{2} & \cdots & \mathbf{x}_{n}
\end{array}\right],
$$

where

$$
\mathbf{x}_{i}=\left[\begin{array}{llll}
x_{1} & x_{2} & \cdots & x_{p}
\end{array}\right]^{T},
$$

i.e. $\mathrm{X}$ contains $n$ measurements of $p$ variables. Compute the covariance matrix $\mathbf{C}_{X}$ as

$$
\mathbf{C}_{X} \approx \frac{1}{n-1}(\mathrm{X}-\overline{\mathrm{X}})(\mathrm{X}-\overline{\mathrm{X}})^{T}
$$

Define similarly another data matrix Y. In the subsequent analysis, $\mathrm{X}$ will refer to a set of measurements for one of the parameters describing vertebral pose and $\mathrm{Y}$ to a set of measurements of another parameter.

For PCA, a linear transform $\mathrm{W}$ is estimated such that the variance of the components of $\mathrm{Z}=\mathrm{W}^{T} \mathrm{X}$ is maximized under the constraint that the components $\mathbf{w}_{i}$ of $\mathrm{W}$ are orthogonal, i.e. the components of $\mathrm{Z}$ are uncorrelated and $\mathbf{C}_{Z}=\mathrm{W}^{T} \mathbf{C}_{X} \mathrm{~W}$ is diagonal. In CCA, two linear transforms, $\mathrm{W}_{X}$ and $\mathrm{W}_{Y}$, are estimated such that the correlation $\rho_{i}$ between the reduced variables (canonical variates) of $\mathrm{W}_{X, i}^{T} \mathrm{X}$ and $\mathrm{W}_{Y, i}^{T} \mathrm{Y}$, have been maximized and that the different components of $\mathrm{W}_{X, i}^{T} \mathrm{X}$ and $\mathrm{W}_{Y, i}^{T} \mathrm{Y}$ are uncorrelated with respect to each other. Note that for CCA, the data matrices $\mathrm{X}$ and $\mathrm{Y}$ are not required to have the same number of variables and, therefore, the number of canonical variates will correspond to the smallest number of variables provided by either X or Y.

An interesting and to us useful aspect of CCA is its relation with mutual information (MI). As shown by [33], the mutual information between $\mathrm{X}$ and $\mathrm{Y}$ can be estimated as the sum of the mutual information of the reduced variables, given that their statistical dependence is limited to correlation. For normally distributed variables, this relation is given as

$$
\mathrm{MI}(\mathrm{X}, \mathrm{Y})=\frac{1}{2} \sum_{i} \log _{2}\left(\frac{1}{\left(1-\rho_{i}^{2}\right)}\right)
$$

The proof for this is given in great detail in [34]. Note that using the log-function with the base 2 provides an MI measure defined in bits. This measure will be employed in the subsequent analysis for quantifying the dependence between different measures. 


\section{Experiments}

To demonstrate the use of the data analysis scheme, measurements of the position and the orientation (i.e. the pose) of the vertebrae for a number of patients were analyzed to determine which of the pair-wise combinations of these measures that have the strongest linear dependence.

\subsection{Image Data}

Image data from 46 patients (35 female and 11 male) were retrospectively gathered and extracted from the local picture archiving and communications system (PACS). The only criteria for inclusion was that the patients suffered from idiopathic scoliosis and that the $\mathrm{CT}$ data had a resolution higher than $1 \times 1 \times 1 \mathrm{~mm}^{3}$. The data sets depicted all lumbar and thoracic vertebrae, i.e. 17 vertebrae per patient. The requirement on the resolution was needed in order to be able to distinguish adjacent vertebrae in the subsequently applied method for obtaining the position and rotation of each vertebra. The patients had an average age of $15.8 \pm 3.0$ years at the time of their respective examinations and an average Cobb angle of 52.2 \pm 12.2 (measured for supine position).

The images were captured as a part of the standard routine for pre-operational planning and they were completely anonymized before being exported from the PACS. Note that for patients of similar age as included in this retrospective study, it is often questionable whether a CT scan is appropriate or not, due to the exposure to radiation. However, at the local hospital there is a protocol in place for acquiring low-dose CT examinations with maintained image quality, targeted towards examinations of the spine. With the use of this protocol, the radiation dose is approximately $0.4 \mathrm{mSv}$. More on this can be found in [35].

\subsection{Vertebral Pose Measures}

Each patient was processed with the method presented by us in [16], which is based on the following steps; extraction of the spinal canal centerline, disc detection, vertebra centerpoint estimation and vertebra rotation estimation. A graphical overview of the method is provided in Figure 3.

1. Spinal canal centerline extraction - Seed points for the spinal canal are detected using the Hough transform in an axial image in the middle of the image volume. A growing and moving circle is used to detect the center of the spinal canal, and where this is repeated for each image as the spinal canal is tracked in both the cranial and the caudal direction.

2. Disc detection - Given that the vertebrae are located anterior to the spinal canal, an intensity profile running through the vertebrae is sampled and filtered, which in turn can be used to detect the position of the discs.

3. Initial vertebral rotation estimation - In the slice between the detected discs, an initial vertebral rotation is estimated based upon minimizing an error measure for assessing the symmetry between two halves of an image.
4. Vertebra centerpoint estimation - The two previous steps provides an initial estimate of the position and the orientation of each vertebra. Based upon analyzing extracted intensity profiles in the anterior-posterior, the rightleft and the caudal-cranial direction, the centerpoint of each vertebra is estimated.

5. Final vertebral rotation estimation - In the last step, the vertebral rotation estimation process is repeated to obtain a final estimate of the rotation of each vertebra.

A more detailed account of the employed method is provided in [16], where the method was shown to have an accuracy of $2.5^{\circ} \pm 2.6$ (mean absolute difference), that was on par with the inter-observer variability for measuring the axial vertebral rotation. This was supported by Bland-Altman plots and high values of the intraclass correlation coefficient $(0.971-0.979)$, thus, showing that the method can be used as a reliable replacement for manual measurements. Since the employed method does not provide identification of each individually detected vertebra, we manually reviewed the detected vertebrae to ensure that measurements of vertebra S1 were not included in the final results. This also allowed us to visually assess the accuracy of the estimated pose of each vertebra.

The method estimates, for each vertebra, the position $[x, y, z]$ and the rotation matrix $\mathrm{R}$, from which the rotation angles $\left[\theta_{X}, \theta_{Y}, \theta_{Z}\right]$ can be derived. The rotation angles were computed as the Euler angles (using a fixed world frame) of the rotation matrix $\mathrm{R}$. Note the order of the rotational angles, $\mathrm{R}=\mathrm{R}_{Z}\left(\theta_{Z}\right) \mathrm{R}_{Y}\left(\theta_{Y}\right) \mathrm{R}_{X}\left(\theta_{X}\right)$ and where $\theta_{Z}$ corresponds to axial rotation, $\theta_{Y}$ to coronal rotation and $\theta_{X}$ to sagittal rotation of the vertebrae. The standard DICOM patient coordinate system was employed to define the orientation of $[x, y, z]$, i.e. $x$ increases from right to left, $y$ from anterior to posterior and $z$ from inferior to superior. Note that the definition employed here for both the patient coordinate system and the rotation angles differ from what has been suggested in [36], where instead $x$ is set to increase from the posterior to anterior and $y$ from left to right. However, the difference in the orientation of the patient coordinate system is merely a difference in notation. The difference in the definition of the rotational angles is much more important to note, where Stokes [36] defines axial vertebral rotation as the angle between the local $y$-axis and global $Y$-axis when projected onto the global axial plane (expressed in accordance with the standard DICOM patient coordinate system). Similarly for the two other rotation angles. The definition proposed in [36] is sensible when investigating the different orientation angles separately but not when analyzing them simultaneously, and leads to very different results, as will be shown later.

Another important aspect to consider is how the patient coordinate system, which is employed to define the position and the rotation of each vertebra, is positioned and oriented in relation to each patient. In our case, we have chosen to center the coordinate system in the centerpoint of vertebra L5. Note that this is performed individually for each patient. The reason for this choice is that we consider vertebra L5 to be the most stable vertebra and, thereby, it forms a suitable anchor point for our measures. One could possibly argue that sacrum or verte- 


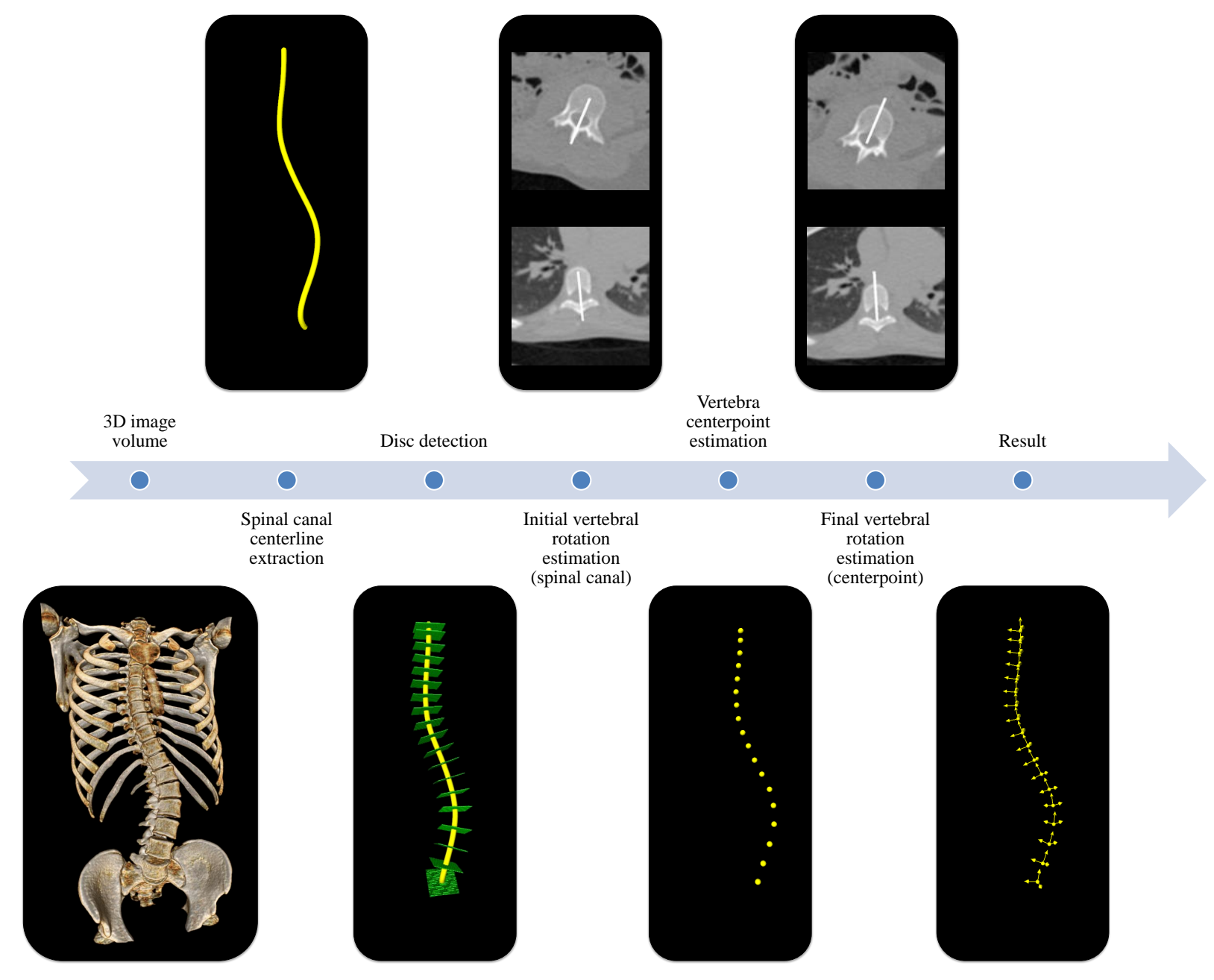

Figure 3: An overview of the method for automatic measurements of the pose of each vertebra in the spine. Note that for illustrative purposes, the input data, i.e. the $3 \mathrm{D}$ image volume, is visualized with volume rendering but the actual processing is done on the actual 3D data volume.

bra $\mathrm{S} 1$ would have been a better choice, but, in this work, this was not an option, since the method employed for obtaining our measurements lack support for determining the position and the rotation of sacrum or S1. The z-coordinates were further normalized such that L5 would have the $\mathrm{Z}$-coordinate 0 and T1 the z-coordinate 1 .

\subsection{Eigenspine Analysis}

In the data analysis, the measurements of each measure for the entire spine were analyzed. The vertebrae indexes were set to define the dimensions of the measurements per curve, i.e. each measure was embedded into a 17 dimensional space. Each measure was then processed with PCA to find its principal components. After the PCA, CCA was applied on the measures projected onto the subspace spanned by the largest PCA eigenvectors of each measure. The CCA was applied to analyze the dependencies between all pair-wise combinations of the different measures.

A reasonable question at this point is why CCA is not applied directly on the estimated measures. The reason for this is two-fold. First, due to the large number of variables (17 vertebrae) compared to the rather low number of observations (46 patients), which can cause singularities in the computations of the CCA, a dimension reduction was called for. Second, using CCA directly is likely to generate an over-fitting, due to its power, i.e. it can find correlations related more to noise in 
Table 1: Canonical correlations and MI as obtained from the pair-wise CCA, sorted according to estimated MI. Here $\rho_{i}$ denotes the correlation between the canonical variates, e.g. between $\mathrm{W}_{X, i}^{T} \mathrm{X}_{y}$ and $\mathrm{W}_{Y, i}^{T} \mathrm{Y}_{\theta_{X}}$ and where $\rho_{i} \geq \rho_{i+1}$. The MI-values are estimated in accordance with (4). Note that the number of estimated canonical correlations are dependent on the number of eigenvectors employed to reconstruct the original measurements.

\begin{tabular}{cccccccccr}
\hline & $\rho_{1}$ & $\rho_{2}$ & $\rho_{3}$ & $\rho_{4}$ & $\rho_{5}$ & $\rho_{6}$ & $\rho_{7}$ & $\rho_{8}$ & MI \\
\hline$x-\theta_{Y}$ & 0.998 & 0.995 & 0.989 & 0.974 & & & & & 12.32 \\
$\theta_{Y}-\theta_{Z}$ & 0.992 & 0.987 & 0.952 & 0.902 & 0.850 & 0.749 & 0.656 & 0.438 & 10.61 \\
$y-\theta_{X}$ & 0.990 & 0.965 & 0.914 & 0.789 & & & & & 6.77 \\
$x-\theta_{Z}$ & 0.987 & 0.976 & 0.943 & 0.568 & & & & & 6.69 \\
$z-\theta_{X}$ & 0.885 & 0.808 & 0.729 & 0.658 & 0.579 & 0.365 & 0.315 & & 3.30 \\
$\theta_{X}-\theta_{Y}$ & 0.865 & 0.734 & 0.695 & 0.638 & 0.604 & 0.465 & 0.338 & 0.158 & 3.01 \\
$\theta_{X}-\theta_{Z}$ & 0.840 & 0.773 & 0.724 & 0.610 & 0.492 & 0.440 & 0.369 & 0.183 & 2.90 \\
$z-\theta_{Y}$ & 0.894 & 0.795 & 0.677 & 0.590 & 0.423 & 0.203 & 0.134 & & 2.81 \\
$z-\theta_{Z}$ & 0.784 & 0.771 & 0.651 & 0.588 & 0.403 & 0.250 & 0.193 & & 2.25 \\
$x-z$ & 0.873 & 0.741 & 0.472 & 0.445 & & & & & 1.95 \\
$x-\theta_{X}$ & 0.831 & 0.661 & 0.629 & 0.413 & & & & & 1.76 \\
$y-z$ & 0.697 & 0.578 & 0.361 & 0.115 & & & & & 0.88 \\
$y-\theta_{Z}$ & 0.634 & 0.565 & 0.409 & 0.262 & & & & & 0.83 \\
$y-\theta_{Y}$ & 0.611 & 0.522 & 0.321 & 0.282 & & & & & 0.70 \\
$x-y$ & 0.525 & 0.358 & 0.115 & 0.064 & & & & & 0.34 \\
\hline
\end{tabular}

the signal than to the relevant variations in the signal, hence, smoothing or a regularization was called for. Both of these requirements can be met by performing a PCA and projecting the signal onto the subspace spanned by the eigenvectors.

\subsection{Stability of Estimated MI Values}

A limiting factor of the performed experiments, discussed in greater detail in the subsequent discussion, is the relatively small number of included measurements in relation to the dimensionality of the measurements, 17 dimensions (vertebrae) for each measurement from 46 patients. Hence, it is of great interest to see how the finally estimated MI values are affected by the number of included patients. To analyze this we performed the same eigenspine analysis as previously described but on a randomly sampled subset of the whole data set. This was repeated 20 times per subset size and where the subset size ranged from 25 to 45 patients.

An important aspect in this analysis is how to determine the number of eigenvectors that should be retained from the PCA and subsequently employed for spanning the subspace onto which the original measurements are projected before the CCA. To properly define this is outside the scope of this paper and, thus, we employ the same approach as in the analysis of the full data set, i.e. eigenvectors sufficient to account for $99 \%$ of the variance in the data were retained.

\section{Results}

In the conducted experiments, PCA was applied to each estimated measure over all patients, followed by a CCA on each pair-wise combination of the measures. From the estimated eigenvalues, the eigenvectors, corresponding to at least $99 \%$ of the variance in the data, were extracted. This meant for instance that the variance in $x$-coordinates could be reduced to four eigenvectors, whereas the variance of $\theta_{X}$ required twelve eigenvectors. Figure 4 depicts the measurements over all measures and patients, and Figure 5 the extracted eigenvectors for each measure. Figure 6 depicts the reconstruction error between the original measurements and the measurements projected onto the subspace of the extracted eigenvectors. In the included figures, the different measurements, the eigenvectors and the reconstruction errors have been plotted using parallel coordinates, a standard approach for visualizing multi-dimensional data. The dimensionality-order of the measurements have been set according to the natural order of the vertebrae in the spine, i.e. vertebra L5 to T1. Note that due to this approach, the data visualized in especially Figure 4 appears to correspond to a spinal curvature as it would be imaged on an anterior-posterior or sagittal radiograph, although the curves in the plot refer to the individual multi-dimensional measurements of a specific measure.

The CCA was applied onto every pair-wise combination of the measures, where the measures were projected onto the subspace spanned by the extracted eigenvectors from the PCA. This was done to find dependencies between the different measures. Table 1 provides the obtained canonical correlations $\rho_{i}$ of all pair-wise CCAs along with corresponding MI estimates. Note the difference in number of canonical correlation coefficients between the different pair-wise comparisons, which is due to the different number of extracted eigenvectors per measure. The results show that the strongest linear dependence exists between the $x$-coordinates and $\theta_{Y}$. A somewhat weaker but still obvious dependence is found between $\theta_{Y}$ and $\theta_{Z}$. A second group of linear relationships, however, substantially weaker than the first two, is found between $y$ and $\theta_{X}$, and between $x$ and $\theta_{Z}$. Or, as expressed in anatomical terms, the lateral displace- 

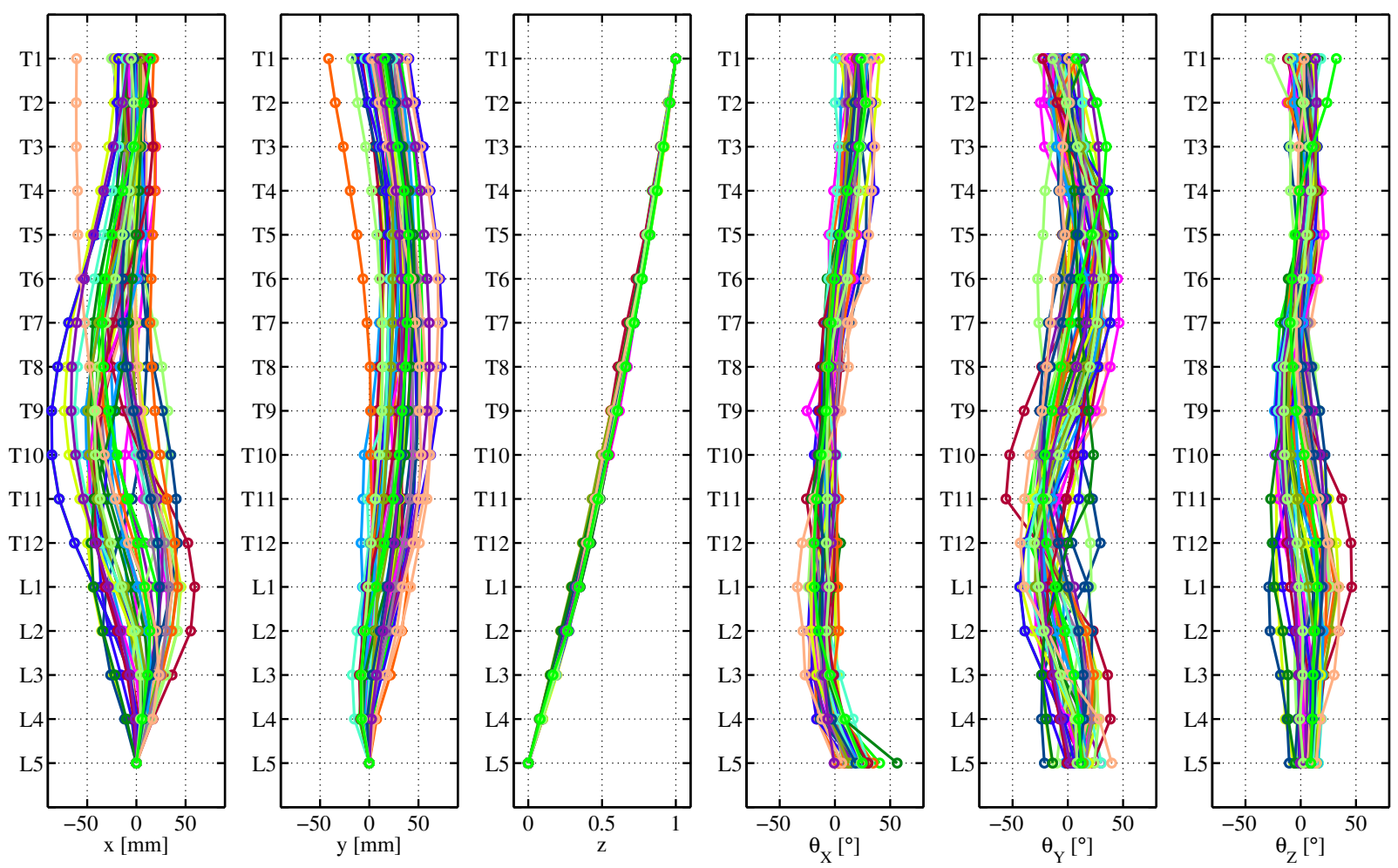

Figure 4: All estimated measurements for all measures and all patients are visualized using parallel coordinates. Note that vertebra L5 serves as origin for all measures, both for positional and rotational measures.
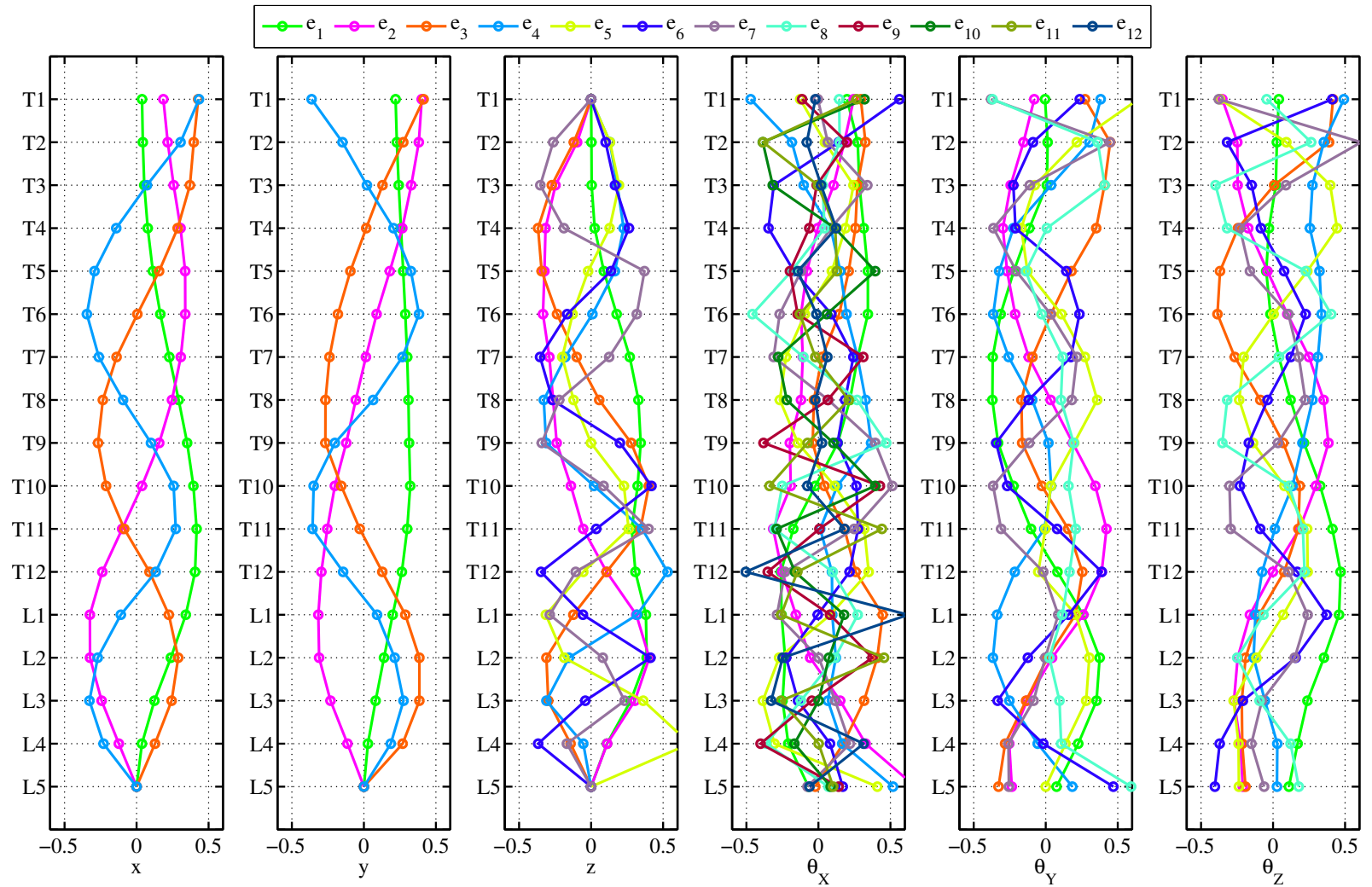

Figure 5: Eigenvectors belonging to the largest eigenvalues as estimated from the PCA, accounting for at least $99 \%$ of the variance in the data. Since visualized using parallel coordinates, the shape of the curves lack a geometrical interpretation. 

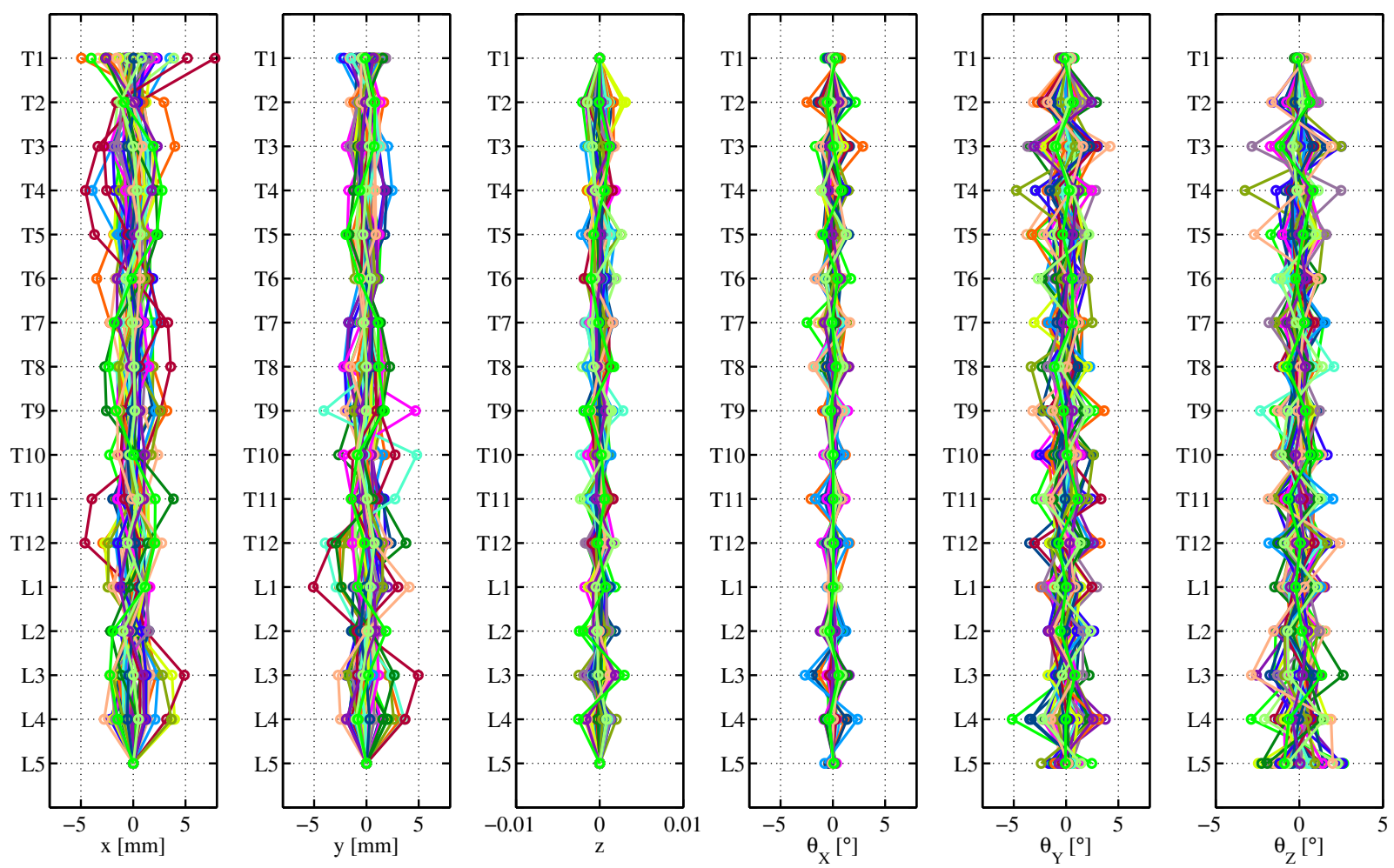

Figure 6: Reconstruction error as given by the difference between the original curves and the curves projected onto the subspace of the eigenvectors accounting for $99 \%$ of the variance in the data. Note that the reconstruction error has been visualized using parallel coordinates.

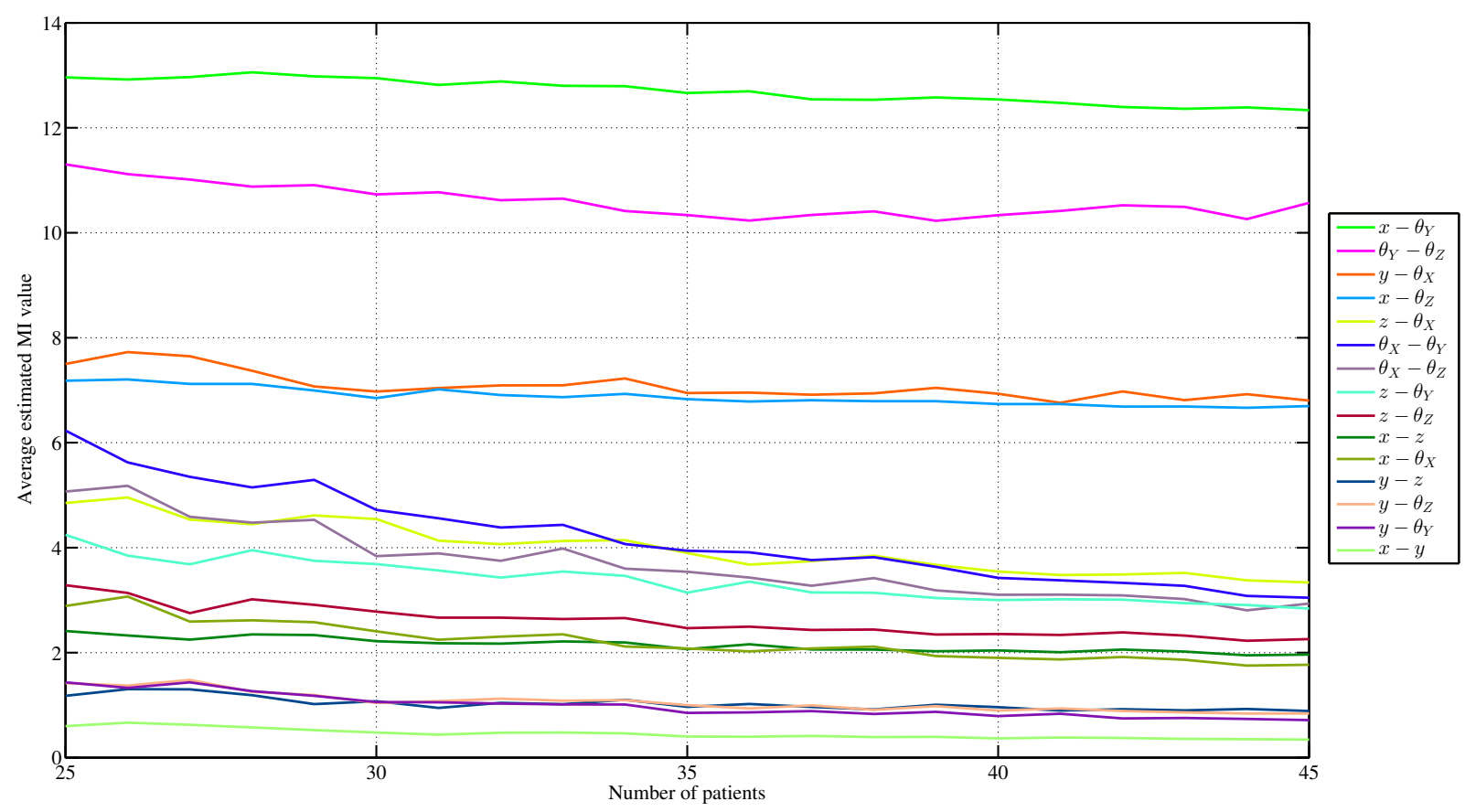

Figure 7: Average estimated MI values as estimated from the eigenspine analysis based upon randomly selected subsets of patients. The random selection and subsequent eigenspine analysis has been repeated 20 times for each subset size ( 25 to 45 patients). 
ment of the vertebral body is highly correlated with the coronal rotation of the same. In addition, the coronal rotation and the axial rotation of the vertebrae are highly correlated. Substantially weaker but still obvious correlations are also found between the lateral displacement and the axial rotation, and between the anterior-posterior displacement and the sagittal rotation.

Figure 7 provides the results from the eigenspine analysis experiments conducted to analyze the stability of the estimated MI-values. Note that the order of the combined measures are the same as in Table 1. Most important to note from the results here is that to a large extent the descending order of the pair-wise combinations does not vary based upon the number of patients included in the eigenspine analysis. In particular the order for the four pair-wise combinations with the strongest correlation does not change at all when varying the number of included patients in the eigenspine analysis. As expected, the magnitude of the estimated MI-values decreases as the number of patients increases.

\section{Discussion}

In this paper, we have presented the concept of eigenspine, a data analysis scheme for determining the relationship between different measures related to spinal deformities. The potential usage of the method has been exemplified by analyzing the dependence between different measures relevant for describing spinal deformities in idiopathic scoliosis. The results of the combined PCA and CCA analysis show, for example, that the strongest linear dependency is found between the lateral displacement and coronal rotation of the vertebral body. That there is a strong linear dependence between these two measures is well in-line with what would be expected and what is previously known. However, more interesting conclusions can be drawn, once the different pair-wise linear dependencies are compared, since this analysis can indicate which measures that are the most relevant for describing a scoliotic curvature.

For instance, the fact that the strongest linear relationship exist between the pair-wise measures $x-\theta_{Y}$ indicates that to describe a scoliotic curvature it is sufficient to either measure the lateral displacement $x$ or the coronal rotation $\theta_{X}$, i.e. both are not needed. Given that there is some linear relationship between axial rotation $\theta_{Z}$ and both coronal rotation $\theta_{Y}$ and lateral displacement $x$, but that it is substantially weaker then the primary correlation, a possible explanation would be that axial rotation $\theta_{Z}$ is a differentiating factor when describing a scoliotic curvature. A conclusion that adds support to the recent interest in quantifying the axial vertebral rotation when assessing idiopathic scoliosis. This further indicates that the classification systems by King and by Lenke [28, 29], are insufficient to fully differentiate between different types of scoliosis, since the axial vertebral rotation is not included in their respective classification systems. Given the limited number of included patients, further analysis including more patients is called for, before any more definite conclusions can be made regarding the order and the strength of the respective pair-wise combinations.
A surprising finding in the presented results is the somewhat weak correlation between the anterior-posterior displacement and the sagittal rotation. In fact, in a preliminary study of ours [37], it was actually the anterior-posterior displacement and the sagittal rotation that showed the strongest correlation. However, here it can be noted that, in the previous work of ours, the range of included scoliosis types were somewhat more constrained than in this work. Further, another normalization of the spatial coordinates was employed and also the rotation measures were derived differently. In fact, if the same spatial normalization and the definition of [36] for computing the rotation measures would be employed on these measurements, the results from the eigenspine analysis would change and provide a correlation between $y-\theta_{X}$ that is almost equally as strong as between $x-\theta_{Y}$, i.e. similar to the results in [37]. Hence, as previously argued, choice of normalization and definition of rotation measures are of importance for the final results.

It is important to point out that the obtained quantification of the linear dependencies between all pair-wise combinations of measures via the computed MI, relies on the assumption of normal distributions for all included variables. This assumption is believed to be reasonable, but further analysis is needed to prove its validity. However, remember that the computation of the MI-values are, in this analysis, simply used as an efficient way of combining the different canonical correlations to a single quantitative measure. In fact, a visual analysis of the estimated canonical correlation for the different pair-wise combinations, as given in Table 1, would provide a similar order and similar understanding of their relative strengths in between each other.

Further, it can be noted that the data analysis scheme has been employed to measures based upon a method [16] for estimating the pose of each vertebra as derived from CT data. However, the eigenspine concept is not limited to these measures or the used method, but could be readily applied to other measurements obtained with e.g. the EOS system. An analysis based upon the data employed in $[25,26,27]$ would be interesting to pursue in order to further quantify the relation between different measures, since the patient groups employed therein are substantially larger than ours. Another interesting future aspect would be to include other measures, e.g. measures related to the deformation that the vertebral bodies undergo during the progression of the scoliotic curvature.

In regards to measures derived from the EOS system, which are based upon the patient in standing position, it is important to point out that the measures used in our case, to determine the pair-wise correlations, are derived from the patient in supine position. This is important to note since a scoliotic curvature will differ for a patient in standing respectively supine position. To what extent the curvature differs depends on, for instance, whether the curvature is functional or structural, where it can be expected that a structural curvature changes less. In addition, it can be expected that the measures of lateral displacement and sagittal rotation will differ more between standing and supine position. To what extent this affects our results is difficult to foresee.

In this work, only the correlation between pair-wise com- 
binations of measures have been analyzed. However, using CCA we believe it would be possible to extend this analysis further, but instead analyzing information content for any number of combined measures by employing autocorrelation. This could be useful to determine a maximally descriptive lowdimensional combination of measures describing spinal deformities in idiopathic scoliosis, and thereby providing means to better relate treatment and outcome of different types of idiopathic scoliosis, which would be a significant clinical outcome.

\section{Conflict of Interest}

The authors declare that there are no conflicts of interest.

\section{Acknowledgments}

The authors would like to thank L. Vavruch and H. Tropp at the Department of Clinical and Experimental Medicine, Linköping University, Sweden, for valuable input in discussions regarding idiopathic scoliosis and for assistance in collecting the used image data. This work was funded by VINNOVA (grant 2012-01213). Visualizations were performed using MeVisLab (provided by Fraunhofer MEVIS, Bremen).

\section{References}

[1] Scoliosis Research Society, SRS Terminology Committee and Working Group on Spinal Classification Revised Glossary of Terms, http://www.srs.org/professionals/glossary/SRS_revised_ glossary_of_terms.htm, 2000.

[2] R. Cobb, Outline for study of scoliosis, American Academy of Orthopaedic Surgeons, Instructional Course Lectures (1948) 261-275.

[3] B. V. Reamy, J. B. Slakey, Adolescent idiopathic scoliosis: review and current concepts, American Family Physician 64 (1) (2001) 111-117.

[4] S. L. Weinstein, L. A. Dolan, J. C. Cheng, A. Danielsson, J. A. Morcuende, Adolescent idiopathic scoliosis, The Lancet 371 (9623) (2008) 1527-1537.

[5] C. R. Templin, J. F. Sarwark, Infantile and juvenile scoliosis, Thieme Medical Publishers Inc., 569-579, 2008.

[6] T. Vrtovec, F. Pernuš, B. Likar, A review of methods for quantitative evaluation of spinal curvature, European Spine Journal 18 (5) (2009) 593607.

[7] W. Skalli, F. Lavaste, J. Descrimes, Quantification of three-dimensional vertebral rotations in scoliosis: what are the true values?, Spine 20 (5) (1995) 546-553.

[8] T. Kuklo, B. Potter, L. Lawrence, Vertebral Rotation and Thoracic Torsion in Adolescent Idiopathic Scoliosis: What is the Best Radiographic Correlate?, Journal of Spinal Disorders and Techniques 18 (2) (2005) 139-147.

[9] B. Heidari, D. Fitzpatrick, D. McCormack, K. Synnott, Correlation of an induced rotation model with the clinical categorization of scoliotic deformity - a possible platform for prediction of scoliosis progression, Stud Health Technol Inform 123 (2006) 169-175.

[10] G. Lam, D. Hill, L. Le, J. Raso, E. Lou, Vertebral rotation measurement: a summary and comparison of common radiographic and CT methods, Scoliosis 3 (1) (2008) 16, ISSN 1748-7161.

[11] T. Vrtovec, F. Pernuš, B. Likar, A review of methods for quantitative evaluation of axial vertebral rotation, European Spine Journal 18 (2009) 1079-1090.

[12] B. P. Rogers, V. M. Haughton, K. Arfanakis, M. E. Meyerand, Application of image registration to measurement of intervertebral rotation in the lumbar spine, Magnetic Resonance in Medicine 48 (6) (2002) 1072-1075.

[13] C. J. Adam, G. N. Askin, Automatic measurements of vertebral rotation in idiopathic scoliosis, Spine 31 (3) (2006) E80-E83.
[14] J.-W. M. Kouwenhoven, K. L. Vincken, L. W. Bartels, R. M. Castelein, Analysis of Pre-existent Vertebral Rotation in the Normal Spine, Spine 31 (13) (2006) 1467-1472.

[15] T. Vrtovec, Modality-Independent Determination of Vertebral Position and Rotation in 3D, in: Medical Imaging and Augmented Reality, vol. 5128 of Lecture Notes in Computer Science, Springer, 89-97, 2008.

[16] D. Forsberg, C. Lundström, M. Andersson, L. Vavruch, H. Tropp, H. Knutsson, Fully automatic measurements of axial vertebral rotation for assessment of spinal deformity in idiopathic scoliosis, Physics in Medicine and Biology 58 (6) (2013) 1775-1787.

[17] R. H. Brown, A. H. Burstein, C. L. Nash, C. C. Schock, Spinal analysis using a three-dimensional radiographic technique, Journal of Biomechanics 9 (6) (1976) 355-IN1.

[18] A. Mitulescu, W. Skalli, D. Mitton, J. De Guise, Three-dimensional surface rendering reconstruction of scoliotic vertebrae using a non stereocorresponding points technique, European Spine Journal 11 (4) (2002) 344-352.

[19] A. Le Bras, S. Laporte, D. Mitton, J. A. De Guise, W. Skalli, Threedimensional 3D detailed reconstruction of human vertebrae from lowdose digital stereoradiography, European Journal of Orthopaedic Surgery \& Traumatology 13 (2) (2003) 57-62.

[20] V. Pomero, D. Mitton, S. Laporte, J. de Guise, W. Skalli, Fast accurate stereoradiographic 3D-reconstruction of the spine using a combined geometric and statistic model, Clinical Biomechanics 19 (3) (2004) 240-247.

[21] J. Dubousset, G. Charpak, I. Dorion, W. Skalli, F. Lavaste, J. Deguise, G. Kalifa, S. Ferey, A new 2D and 3D imaging approach to musculoskeletal physiology and pathology with low-dose radiation and the standing position: the EOS system, Bulletin de l'Academie nationale de medecine 189 (2) (2005) 287.

[22] T. Easwar, J. Hong, J. Yang, S. Suh, H. Modi, Does lateral vertebral translation correspond to Cobb angle and relate in the same way to axial vertebral rotation and rib hump index? A radiographic analysis on idiopathic scoliosis, European Spine Journal 20 (7) (2011) 1095-1105.

[23] J. Y. Hong, S. W. Suh, T. R. Easwar, H. N. Modi, J. H. Yang, J. H. Park, Evaluation of the Three-Dimensional Deformities in Scoliosis Surgery With Computed Tomography: Efficacy and Relationship With Clinical Outcomes, Spine 36 (19) (2011) E1259-E1265.

[24] F. J. Schwab, V. A. Smith, M. Biserni, L. Gamez, J. P. C. Farcy, M. Pagala, Adult scoliosis: a quantitative radiographic and clinical analysis, Spine 27 (4) (2002) 387-392.

[25] L. Duong, F. Cheriet, H. Labelle, Three-dimensional classification of spinal deformities using fuzzy clustering, Spine 31 (8) (2006) 923-930.

[26] S. Kadoury, H. Labelle, Classification of three-dimensional thoracic deformities in adolescent idiopathic scoliosis from a multivariate analysis, European Spine Journal 21 (1) (2012) 40-49.

[27] A. P. Sangole, C. E. Aubin, H. Labelle, I. A. F. Stokes, L. G. Lenke, R. Jackson, P. Newton, Three-dimensional classification of thoracic scoliotic curves, Spine 34 (1) (2008) 91-99.

[28] H. A. King, J. H. Moe, D. S. Bradford, R. B. Winter, The selection of fusion levels in thoracic idiopathic scoliosis, J Bone Joint Surg Am 65 (9) (1983) 1302-13.

[29] L. G. Lenke, R. R. Betz, J. Harms, K. H. Bridwell, D. H. Clements, T. G. Lowe, K. Blanke, Adolescent idiopathic scoliosis a new classification to determine extent of spinal arthrodesis, The Journal of Bone \& Joint Surgery 83 (8) (2001) 1169-1181.

[30] S. Kadoury, J. Shen, S. Parent, Global geometric torsion estimation in adolescent idiopathic scoliosis, Medical \& Biological Engineering \& Computing (2013) 1-11.

[31] K. Pearson, On lines and planes of closest fit to systems of points in space, The London, Edinburgh, and Dublin Philosophical Magazine and Journal of Science 2 (11) (1901) 559-572.

[32] H. Hotelling, Relations between two sets of variates, Biometrika 28 (3-4) (1936) 321-377.

[33] J. Kay, Feature discovery under contextual supervision using mutual information, in: Neural Networks, 1992. IJCNN., International Joint Conference on, vol. 4, IEEE, 79-84, 1992.

[34] M. Borga, Learning Multidimensional Signal Processing, Linkping Studies in Science and Technology. Dissertations No. 531, Linkping University, Sweden, 1998.

[35] M. K. Kalra, P. Quick, S. Singh, M. Sandborg, A. Persson, Whole spine CT for evaluation of scoliosis in children: feasibility of sub-milliSievert scanning protocol., Acta Radiologica 54 (2) (2013) 226-230. 
[36] I. A. F. Stokes, Three-dimensional terminology of spinal deformity: a report presented to the Scoliosis Research Society by the Scoliosis Research Society Working Group on 3-D terminology of spinal deformity, Spine 19 (2) (1994) 236-248.
[37] D. Forsberg, C. Lundström, H. Knutsson, Eigenspine: Eigenvector Analysis of Spinal Deformities in Idiopathic Scoliosis, in: A. Heyden, F. Kahl (Eds.), Spinal Imaging and Image Analysis, Lecture Notes in Computational Vision and Biomechanics, Springer Heidelberg, 2014. 